\title{
RANGE EXTENSION OF BRACHYURAN CRABS ALONG THE BAJA CALIFORNIA COAST, MEXICO (CRUSTACEA: DECAPODA)
}

\section{EXTENSIÓN DEL ÁMBITO GEOGRÁFICO DE CANGREJOS BRAQUIUROS A LO LARGO DE LA COSTA DE BAJA CALIFORNIA, MÉXICO (CRUSTACEA: DECAPODA)}

\author{
Ernesto Campos* \\ Gerardo López \\ Facultad de Ciencias \\ Universidad Autónoma de Baja California \\ Apartado postal 2300 \\ Ensenada, CP 22800, Baja California, México \\ *E-mail: ecampos@bahia.ens.uabc.mx
}

Recibido en septiembre de 1997; aceptado en diciembre de 1997

\begin{abstract}
The study of brachyuran crabs collected from intertidal areas of the upper Gulf of California and the west coast of the Baja California peninsula, at Tortugas Bay, Baja California Sur, has produced a northern range extension for the following species: Hemus finneganae Garth, 1958; Microphrys platysoma (Stimpson, 1860); Eriphia squamata Stimpson, 1859; Xanthodius sternberghii Stimpson, 1859; Uca princeps princeps (Smith, 1870); and Euphylax robustus A. Milne-Edwards, 1874. We present each species and include an analysis of their previous distribution, habitat, new localities and, in some cases, notes on the breeding period.
\end{abstract}

Key words: Crustacea, Brachyura, distribution, Baja California.

\section{RESUMEN}

El estudio de cangrejos braquiuros recolectados en las áreas intermareales del alto Golfo de California y en la costa occidental de la península de Baja California, en Bahía Tortugas, Baja California Sur, ha originado una extensión del ámbito geográfico norteño para las siguientes especies: Hemus finneganae Garth, 1958; Microphrys platysoma (Stimpson, 1860); Eriphia squamata Stimpson, 1859; Xanthodius sternberghii Stimpson, 1859; Uca princeps princeps (Smith, 1870); y Euphylax robustus A. Milne-Edwards, 1874. Presentamos a cada especie e incluimos un análisis sobre su distribución previa, su hábitat, nuevas localidades $\mathrm{y}$, en algunos casos, notas sobre el periodo reproductivo.

Palabras clave: Crustacea, Brachyura, distribución, Baja California.

\section{INTRODUCTION}

The study of brachyuran crabs collected from intertidal areas in the Gulf of California and along the west coast of the Baja California peninsula, at Tortugas Bay, Baja California Sur,

\section{INTRODUCCIÓN}

El estudio de cangrejos braquiuros recolectados en el intermareal del Golfo de California y sobre la costa occidental de la península de Baja California, en Bahia Tortugas, Baja 
allows us to report a northern range extension for 6 species (2 Majidae, 1 Menippidae, 1 Ocypodidae. 1 Portunidae and 1 Xanthidae). The new records provided herein update those of Hendrickx (1995). The following abbreviations are used: Gulf of California, GC; Baja California. BC; Baja California Sur, BCS; Sonora, SON.

\section{MATERIAL AND METHODS}

During the last 10 years, brachyuran crabs have been collected by hand during low tides in the rocky intertidal and sandy shores at several localities along the Mexican Pacific. These include: Puerto Peñasco, SON; San Felipe, BC; Puertecitos, BC; Los Ángeles Bay, BC; estuary of the Mulegé River. BCS; Concepción Bay, $\mathrm{BCS}$; and Bahía Tortugas, on the west coast of the $\mathrm{BC}$ peninsula. The specimens collected were placed in plastic bags and submerged in cold water. Later, they were fixed and preserved in ethanol or 2 -propanol $(70 \%)$. The material is deposited in the Laboratorio de Invertebrados. Facultad de Ciencias, Universidad Autónoma de Baja California.

\section{RESULTS AND DISCUSSION}

\section{Family Majidae \\ Hemus finneganae Garth, 1958}

Previous distribution: From Ángel de la Guarda Island, BC, and Puerto San Carlos, SON, GC, Mexico, to Santa Elena Bay, Ecuador, including the Revillagigedo Islands (Garth, 1958).

Material examined: 2 males, 3 ovigerous females, Puertecitos, BC, April-May 1986.

Remarks: The present record extends the range of II. finneganae about $135 \mathrm{~km}$ north along the east coast of the $\mathrm{BC}$ peninsula. The crabs were collected under rocks, among algae, sponges, tubicolous polychactes and bryozoans. The carapaces were covered with aigae and sponges. Ovigerous females were collected in May; they have previously been recorded in January and February in the tropical Pacific (Garth. 1958).
California Sur, nos permite reportar una extensión norteña del ámbito geográfico para 6 especies (2 Majidae, 1 Menippidae, 1 Ocypodidac, 1 Portunidae y 1 Xanthidae). Los nuevos registros aquí otorgados actualizan aquellos de Hendrickx (1995). Se utilizan las siguientes abreviaturas: Golfo de California, GC; Baja California, BC: Baja California Sur, BCS; Sonora, SON.

\section{MATERIAL Y MÉTODOS}

Durante los últimos 10 años, en mareas vivas, especies de cangrejos braquiuros han sido recolectados manualmente en el intermareal rocoso y playas arenosas de varias localidades del Pacífico mexicano. Éstas incluyen: Puerto Peñasco, SON; San Felipe, BC; Puertecitos, BC; Bahía de los Ángeles, BC; estuario del Río Mulegé, BCS; Bahía Concepción, BCS; y Bahía Tortugas, sobre la costa occidental de la península de BC. Los especímenes recolectados fueron colocados en bolsas de plástico y sumergidos en agua helada. Luego fueron fijados y preservados en etanol ó 2-propanol al $70 \%$. El material está depositado en el Laboratorio de Invertebrados, Facultad de Ciencias, Universidad Autónoma de Baja California.

\section{RESULTADOS Y DISCUSIÓN}

\section{Familia Majidae \\ Hemus finneganae Garth, 1958}

Distribución previa: Desde Isla Ángel de la Guarda, BC, y Puerto San Carlos. SON, GC, México, hasta Bahia de Santa Elena, Ecuador, incluyendo las islas Revillagigedo (Garth, 1958).

Material examinado: 2 machos, 2 hembras ovigeras, Puertecitos, BC, abril-mayo de 1986.

Observaciones: El presente registro extiende el ámbito de $H$. finneganae cerca de $135 \mathrm{~km}$ al norte sobre la costa oriental de la península de $B C$. Los cangrejos fueron recolectados bajo rocas, entre algas, esponjas, poliquetos tubícolos y briozoarios. El caparazón estuvo cubierto 


\section{Family Majidae}

Microphrys platysoma (Stimpson, 1860)

Previous distribution: From Puerto Peñasco, SON, and Puerto Refugio, Ángel de la Guarda Island, $\mathrm{BC}, \mathrm{GC}, \mathrm{Mexico}$, to Santa Elena Point, Ecuador, including Socorro Island (Garth, 1958; Vogel, 1966).

Material examined: 1 male, 3 females ( 2 ovigerous), Puertecitos, BC, May-June 1986.

Remarks: The present record extends the range of $M$. platysoma about $135 \mathrm{~km}$ north of its previous northernmost limit along the east coast of the $\mathrm{BC}$ peninsula. This species was collected under rocks, among algae and sponges that covered their carapaces. Ovigerous females were collected in May and June; previously recorded from February to August in the GC (Garth, 1958).

\section{Family Menippidae}

Eriphia squamata Stimpson, 1859

Previous distribution: From Puerto Peñasco, SON, and San Felipe, BC, GC, Mexico, to Las Vacas near Capón, Peru; west coast of $\mathrm{BC}$ peninsula to San Juanico Bay, BCS (Garth, $1957,1960)$.

Material examined: 1 female, La Bajada Beach, Tortugas Bay, BCS, 15 October 1993.

Remarks: The present record extends the range of E. squamata about $370 \mathrm{~km}$ north of its previous northernmost limit on the west coast of the $\mathrm{BC}$ peninsula. It is a rare species in Tortugas Bay. Collecting efforts during the last five years produced only one female. It was collected under rocks at a protected rocky beach, consisting of a mixture of sand, mud, gravel and pebbles.

\section{Family Xanthidae}

Xanthodius sternberghii Stimpson, 1859

Previous distribution: From Puerto Lobos, Guaymas, SON, and Mulegé, BCS, GC, to Isla con algas y esponjas. Hembras ovigeras fueron recolectadas en mayo; previamente han sido registradas en enero y febrero en el Pacífico tropical (Garth, 1958).

\section{Familia Majidae}

Microphrys platysoma (Stimpson, 1860)

Distribución previa: Desde Puerto Peñasco, SON, y Puerto Refugio, Isla Ángel de la Guarda, BC, GC, México, hasta Punta Santa Elena, Ecuador, incluyendo Isla Socorro (Garth, 1958; Vogel, 1966).

Material examinado: 1 macho, 3 hembras (2 ovígeras), Puertecitos, $\mathrm{BC}$, mayo-junio de 1986.

Observaciones: El presente registro extiende el ámbito de $M$. platysoma cerca de $135 \mathrm{~km}$ al norte de su límite norteño previo sobre la costa oriental de la peninsula de BC. Esta especie fue recolectada bajo rocas, entre algas y esponjas, las cuales cubrian su caparazón. Se recolectaron hembras ovígeras en mayo y junio; previamente éstas fueron registradas de febrero a agosto en el GC (Garth, 1958).

\section{Familia Menippidae \\ Eriphia squamata Stimpson, 1859}

Distribución previa: Desde Puerto Peñasco, SON, y San Felipe, BC, GC, México, hasta Las Vacas, cerca de Capón, Perú; costa occidental de la península de $\mathrm{BC}$ hasta Bahía San Juanico, BCS (Garth, 1957, 1960).

Material examinado: 1 hembra, playa La Bajada, Bahía Tortugas, BCS, 15 de octubre de 1993.

Observaciones: El presente registro extiende el ámbito de distribución de E. squamata cerca de $370 \mathrm{~km}$ al norte de su límite norteño previo sobre la costa occidental de la península de BC. Ésta es una especie rara en Bahía Tortugas. Recolectas durante los últimos cinco años produjeron una sola hembra. Ésta fue recolectada bajo rocas, en una playa rocosa protegida que 
María Madre, off Nayarit, and west coast of $\mathrm{BC}$ peninsula to Magdalena Bay, BCS, Mexico (Villalobos-Hiriart et al., 1989; Hendrickx, 1995).

Material examined: 2 males, 2 females, San Felipe and vicinity, BC, 1988-1995; 2 males, 3 females, Puertecitos, BC, July-September 1989.

Remarks: The present record extends the range of $X$. sternberghii about $600 \mathrm{~km}$ north along the east coast of the $\mathrm{BC}$ peninsula. It lives under rocks on a sandy gravel bottom. Based on carapace morphology, Crane (1947) concluded that $X$ hebes Stimpson, 1860, should be considered a junior synonym of $X$ sternberghii. However, Guinot (1967) does not concur with this statement. Except for Brusca (1980), who follows Guinot's recommendation, others have assumed Crane's conclusion (see Villalobos-Hiriart et al. 1989; Hendrickx, 1995). We have followed, with hesitation, Crane's (1947) recommendation, since we consider that a detailed morphological study of variation in gonopod shape is necessary to resolve the taxonomic status of these species.

\section{Family Ocypodidae}

Uca princeps princeps (Smith, 1870)

Previous distribution: From Rasa Island (GC) and west coast of $\mathrm{BC}$ peninsula at 'Tortugas Bay, BCS (= Bartolomé Bay), Mexico, to Zamurilla River, Peru (Crane, 1975; VillalobosHiriart et al., 1989).

Material examined: 10 males and 10 females, Laguna Percebú, San Felipe, BC, July-August 1995.

Remarks: This is the first record of $U . p$. princeps for the peninsular coast of the GC. It extends the range about $390 \mathrm{~km}$ north of its previous northernmost limit in the GC. This species inhabits a salt-marsh area south of Laguna Percebú Ranch. consiste de una mezcla de arena, lodo, grava y pedruscos.

\section{Familia Xanthidae}

Xanthodius sternberghii Stimpson, 1859

Distribución previa: Desde Puerto Lobos, Guaymas, SON, y Mulegé, BCS, GC, hasta la Isla María Madre, cerca de Nayarit; costa occidental de la península de BC hasta Bahía Magdalena, BCS, México (Villalobos-Hiriart et al., 1989; Hendrickx, 1995).

Material examinado: 2 machos, 2 hembras, San Felipe y sus vecindades, BC. 1988-1995; 2 machos, 3 hembras, Puertecitos, BC, julioseptiembre de 1989

Observaciones: El presente registro extiende al ámbito norteño de $X$. sternberghii cerca de $600 \mathrm{~km}$ a lo largo de la costa oriental de la península de BC. Este cangrejo vive bajo rocas, sobre un fondo de grava arenosa. Con base en la morfología del caparazón, Crane (1947) concluyó que $X$. hebes (Stimpson 1960) debería ser considerado un sinónimo reciente de $X$. sternberghii. Guinot (1967) no concurrió con tal afirmación. A excepción de Brusca (1980), quien siguió la recomendación de Guinot, otros han asumido la conclusión de Crane (ver Villalobos-Hiriart et al, 1989; Hendrickx, 1995). Nosotros hemos seguido, con vacilación, la recomendación de Crane (1947), ya que consideramos que un estudio morfológico detallado sobre la variación del gonópodo es necesario para resolver el estatus taxonómico de estas especies.

\section{Familia Ocypodidae}

Uca princeps princeps (Smith, 1870)

Distribución previa: Desde Isla Rasa, GC, y costa occidental de la península de $\mathrm{BC}$ en Bahía Tortugas, BCS (= Bahía Bartolomé), México, hasta Río Zamurilla, Perú (Crane, 1975; Villalobos-Hiriart et al., 1989).

Matcrial cxaminado: 10 machos, 10 hembras, Laguna Percebú, San Felipe, BC, julio-agosto de 1995. 


\section{Family Portunidae}

Euphylax robustus A. Milne-Edwards, 1874

Previous distribution: From Santa Clara, SON (GC), Mexico, to Paita, Peru (Garth and Stephenson, 1966; Brusca, 1980).

Material examined: 1 male, La Bajada Beach, Tortugas Bay, BCS, October 1993.

Remarks: This is the first record of this species on the west coast of the $\mathrm{BC}$ peninsula. The male reported herein was dying when collected in a small tidal pool.

\section{General remarks}

The brachyuran crabs are the largest group of decapod crustaceans in the Mexican Pacific (Hendrickx, 1993, 1995). Although monumental revisions and detailed checklists on this group have been published (see Garth, 1960; Hendrickx, 1995), modern studies in this area (see literature cited in IIendrickx, 1995) support the fact that a critical analysis on taxonomy and ecological factors related to the adult distribution are still needed for many species. We consider that such kind of studies are necessary for at least three groups of species: (1) small, cryptic and taxonomically problematic species (e.g., Pinnotheridae); (2) species with a peculiar or restricted habitat (e.g., Grapsidae-Varuninae); and (3) fairly common species that have never been properly studied from a taxonomic viewpoint (e.g., Pilumnidae). In addition, we consider it important to recognize: (1) that the fast coastal cxpansion is degrading diverse habitats along the continental and peninsular coasts of the Mexican Pacific, and (2) that marked ecological differences exist between the habitats along the continental and peninsular coasts. In this context, we consider that complementary records of species and habitats are highly recommendable to complete the distributional patterns of the brachyuran crabs along the $\mathrm{GC}$ and west coast of the $\mathrm{BC}$ peninsula.
Observaciones: Éste es el primer registro de $U$. p. princeps para la costa peninsular del GC. Su ámbito norteño se extiende cerca de $390 \mathrm{~km}$ de su límite previo en el GC. Esta especie habita un área de saladares al sur del Rancho Laguna Percebú.

\section{Familia Portunidae}

Euphylax robustus A. Milne-Edwards, 1874

Distribución previa: Desde Santa Clara, SON, GC, México, hasta Paita, Perú (Garth y Stephenson, 1966; Brusca, 1980).

Material examinado: 1 macho, playa La Bajada, Bahía Tortugas, BCS, octubre de 1993.

Observaciones: Éste es el primer registro de $E$. robustus para la costa occidental de la península de BC. El macho aquí registrado estaba moribundo cuando se le recolectó en una pequeña poza de marea.

\section{Observaciones generales}

Los cangrcjos braquiuros son cl grupo más numeroso de crustáceos decápodos en el Pacífico mexicano (Hendrickx, 1993, 1995). Aunque amplias revisiones y detalladas listas de especies sobre este grupo han sido publicadas (ver Garth, 1948; Hendrickx, 1995), estudios modernos en esta área (ver literatura citada en Hendrickx, 1995) apoyan el hecho de que un análisis crítico sobre taxonomía y factores ecológicos relacionados con la distribución de adultos son aún necesarios para muchas especies. Nosotros consideramos que tales estudios son necesarios para al menos tres grupos de especies: (1) las pequeñas, cripticas y taxonómicamente difíciles (e.g., Pinnotheridae); (2) aquellas con un hábitat peculiar o restringido (e.g., Grapsidae-Varuninae); y (3) las comunes que nunca han sido adecuadamente estudiadas desde un punto de vista taxonómico (e.g., Pilumnidae). En conexión, consideramos importante reconocer: (1) que la rápida expansión está degradando diversos hábitats a lo largo de la costa peninsular y continental del Pacífico mexicano; y (2) que marcadas diferencias ecológicas existen entre los hábitats a lo 


\section{ACKNOWLEDGEMENTS}

This work was supported by the project UABC-0134 "Crustáceos simbiontes del Pacifico mexicano" of the Facultad de Ciencias, Universidad Autónoma de Baja California (UABC), and agreement UABC-CONACYT 3587-N9311. The first author is a fellow of the "Programa de Estímulo al Personal Académico de la UABC 95/97".

\section{REFERENCES}

Brusca, R.C. (1980). Common Intertidal Invertebrates of the Gulf of California. Univ. Arizona Press, Tucson, $513 \mathrm{pp}$.

Crane, J. (1947). Eastern Pacific Expedition of the New York Zoological Society. XXXVIII. Intertidal brachygnathous crabs from the west coast of tropical America with special reference to ecology. Zoologica, 32(9): 69-95.

Crane, J. (1975). Fiddler Crabs of the World. Ocypodidae: Genus Uca. Princeton Univ. Press, Princeton, New Jersey, $736 \mathrm{pp}$.

Garth, J.S. (1957). The Crustacean Decapoda Brachyura of Chile. The Lund University Chile Expedition 1948-49. Lunds Universitets Arsskrift, N.F. Avd. 2, Bd. 53, Nr. 7: 1-127.

Garth, J.S. (1958). Brachyura of the Pacific coast of America. Oxyrhyncha. Allan Hancock Pac. Exped., 21: 1-854.

Garth, J.S. (1960). Distribution and affinities of the brachyuran Crustacea. (Symposium: The biogeography of Baja California and adjacent sea. Part II. Marine biotas.) Syst. Zool., 9: 105-123.

Garth, J.S. and Stephenson, W. (1966). Brachyura of the Pacific coast of America. Brachyrhyncha: Portunidae. Allan Hancock Monog. Mar. Biol, 1: $1-154$.

Guinot, D. (1967). Recherches préliminaires sur les grupements naturels chez les crustacés décapodes brachyoures. IV. Observations sur quelques generes de Xanthidae. Bull. Mus. Nat. Hist. nat. $2^{\mathrm{e}}$ Ser, 39(4): 695-727. largo de la costa continental y peninsular. En este contexto, consideramos que registros complementarios de especies y hábitats es altamente recomendable para completar los patrones de distribución de los cangrejos braquiuros (y otros invertebrados) a lo largo del GC y la costa occidental de la península de BC.

\section{AGRADECIMIENTOS}

Este trabajo fue apoyado por el proyecto UABC-0134 "Crustáceos simbiontes del Pacifico mexicano" de la Facultad de Ciencias, Universidad Autónoma de Baja California (UABC), y convenio UABC-CONACYT 3587N9311. El primer autor es un becario en el "Programa de Estímulo al Personal Académico de la Universidad Autónoma de Baja California 95/97".

Traducido al español por el autor.

Hendrickx, M.E. (1993). Crustáceos decápodos del Pacífico mexicano. En: S.I. Salazar-Vallejo y N.E. González (eds.), Biodiversidad Marina y Costera de México. CONABIO-CIQRO, México, pp. 271-318.

Hendrickx, M.E. (1995). Checklist of brachyuran crabs (Crustacea: Decapoda) from the eastern tropical Pacific. Bull. Inst. Roy. Sci. Nat. Belgique (Biologie), 65: 125-150.

Villalobos-Hiriart, J.L., Nates-Rodríguez, J.C., Cantú-Diaz-Barriga, A., Valle-Martínez, M.D., Flores-Hernández, P., Lira-Fernández, E. y Schmidtsdorf-Valencia, P. (1989). Listados Faunísticos de México. I. Crustáceos estomatópodos y decápodos intermareales de las islas del Golfo de California, México. Inst. de Biologia, Univ. Nacional Autónoma de México, 114 pp.

Vogel, B.R. (1966). A report on a collection of crabs from the Gulf of California. Southwest. Nat., 11(1): 139-140. 